\title{
L'açaí en Amazonie : la diversité des goûts au cœur de la coexistence de circuits courts et globaux
}

\author{
Nathalie CIALDELLA • Cirad, UMR Innovation, Guyane française \\ nathalie.cialdella@cirad.fr \\ Edfranklin SILVA • Université fédérale du Pará, Matinha, Cametá, Brésil \\ Livia NAVEGANTES-ALVES • Université fédérale du Pará, Guamá, Belém, Brésil \\ Inavegantes@ufpa.br \\ Janaina DEANE DE ABREU SÁ DINIZ • Université de Brasilia, Planaltina, Brésil \\ janadiniz@unb.br
}

En Amazonie brésilienne, la pulpe d'açaí se consomme traditionnellement naturelle et accompagne des plats salés. Depuis les années 1990, I'açaí s'exporte sur des modes de consommation sucrés. Les auteurs s'interrogent sur le rôle des modes de consommation et des mécanismes qui garantissent la " qualité " aux consommateurs, dans la coexistence de différents circuits de commercialisation de la région métropolitaine de Belém (Pará). La manière dont les transformateurs traitent de la qualité renvoie à des attributs variés : sanitaire, productive (bio), sociale (équitable), gustative. Mise en avant exclusivement dans les circuits courts et non standardisée, la qualité gustative est liée à l'origine du fruit, aux pratiques de cueillette, de production et de transport. Circuits longs et courts se distinguent par les goûts des consommateurs et par des mécanismes formels ou informels de certification, basés sur des relations de confiance. Leur coexistence future dépend de la mise en place de mécanismes garantissant la qualité gustative des pulpes, la diversité des goûts des consommateurs et l'inclusion des populations traditionnelles dans la filière.

MOTS-CLÉS : qualité, goût, açaí, coexistence, circuits courts et globaux

\section{Açaí in the Amazon: diversity of tastes at the core of the coexistence of short and global chains}

IIn the Brazilian Amazon, people traditionally consume açaí pulp natural and with salty meals. Since the 1990s, açaí has been exported with sweeter modes of consumption. The authors consider the role of consumption patterns and mechanisms that guarantee the "quality" to consumers, in the coexistence of different chains in the metropolitan area of Belém (Pará). The way in which processors deal with quality, refers to various characteristics: sanitary, productive (organic), social (fair), taste. Exclusively promoted in short chains but not yet standardized, taste quality is linked to the origin of the fruit, to the gathering, production and transport practices. Global versus short differs in terms of consumer tastes and formal certification schemes versus informal mechanisms based on trust relationships. Their future coexistence depends on the implementation of mechanisms that guarantee the taste quality of the pulp, the diversity of consumers' tastes and the inclusion of traditional populations in the sector. (JEL: 013, Q23, R32).

KEYWORDS: quality, taste, açaí, coexistence, short and global food supply chains 


\section{La fièvre de l'açaí et ses impacts au niveau local}

Consommer la pulpe d'açaí, obtenue à partir de l'extraction de la baie du palmier açaí (Euterpe oleracea Mart. ${ }^{1}$ ), est une tradition culinaire partagée par l'ensemble de la population du bassin amazonien. Le partage de cette tradition pourrait venir de la large distribution de l'espèce dans tout l'estuaire amazonien (Azevedo et Kato, 2007) ainsi que des propriétés nutritionnelles du fruit (Rogez, 2000 ; Béreau, 2001). La pulpe d'açaí se consomme localement naturelle, mélangée ou non à de la farine de manioc, accompagnant des plats de poisson frit, de crevettes ou de bœuf salé-séché (charque). Le développement économique de la filière açaí a démarré dans les années 1970 avec l'arrivée de populations rurales modestes dans les centres urbains régionaux (Brondizio et al., 2002). À partir du milieu des années 1990 démarre la «fièvre de l'açaí ». Depuis, le marché est en expansion constante, avec une augmentation de la demande caractérisée par l'inélasticité des prix (Homma et al., 2006 ; Nogueira et al., 2013). L'État du Pará produit près de $90 \%$ de l'açaí total venant d'Amazonie et concentre entre $60 \%$ et $80 \%$ de la consommation de pulpe (selon les sources et les années), ainsi que la plupart des marchés de gros et des industries transformatrices du pays. La pulpe s'exporte vers les États du Sud et Centre-Sud du Brésil, ainsi qu'à l'international (Japon, ÉtatsUnis, Europe principalement) sur d'autres modes de consommation : sucré et mixé à d'autres fruits (banane, fraise, guarana) pour en faire des sorbets, des yaourts, etc. Ces nouveaux modes de consommation s'appuient sur des propriétés vertueuses de l'açaí (présence d'antioxydants) et

1. Voir en particulier les travaux de Jardim et Anderson du Museu Paraense Emilio Goeldi sur l'évolution des modes de gestion et des usages de ce palmier. non sur les critères gustatifs, visuels et de texture, mis en avant par les consommateurs amazoniens. Le profil des consommateurs s'est ainsi diversifié. Les classes économiquement modestes, voire marginales d'Amazonie, ayant pour habitude de consommer quotidiennement l'açaí pendant la saison principale de cueillette maintiennent leurs pratiques malgré l'augmentation des prix (Brondizio et al., 2002 ; Nogueira et al., 2013). À cette catégorie de consommateurs s'ajoutent aujourd'hui les classes moyennes brésiliennes et étrangères, attirées par l'açaí en tant que « super-aliment» ou aliment fonctionnel (Menezes et al., 2011).

La fièvre de l'açaí coïncide à ses débuts avec un moment historique en Amazonie, où le gouvernement brésilien entérine les mouvements socio-environnementaux par la reconnaissance des populations indigènes et afrodescendantes (quilombolas) dans sa Constitution de 1988, puis des populations extractivistes avec la création des réserves extractivistes en $1990^{2}$. L'expansion du marché de l'açaí touche directement ces populations et les populations ribeirinhas habitant sur les marges des fleuves. La vente du fruit d'açaí compose alors jusqu'à $70 \%$ du revenu de ces familles pendant la période de cueillette (Brondizio, 2008), bouleversant, souvent de manière invisible, les modes de vie (Steward, 2013). En 2007, le gouvernement reconnaît l'existence spécifique des ribeirinhos, en adoptant de manière plus large la notion de Peuples et communautés traditionnelles $(\mathrm{PCT})^{3}$, que nous simplifierons en utilisant le terme de

2. «Espaces territoriaux destinés à l'exploration durable et la conservation des ressources naturelles renouvelables par les populations extractivistes » (décret 98.897, du 30 janvier 1990).

3. «Groupes culturellement différenciés et qui se reconnaissent comme tels, possèdent des formes propres d'organisation sociale, occupent et utilisent des territoires et ressources naturelles comme condition de leur reproduction [...]» (décret 6.040, du 7 février 2007). 
population traditionnelle. Dans les années 2000, l'omniprésence des populations traditionnelles en tant que fournisseurs de fruits d'açaí est perturbée par l'arrivée de nouveaux acteurs. Par son attractivité économique et son caractère endémique, le palmier açaí devient une des espèces phares dans la restauration des couverts forestiers et attire des agriculteurs-éleveurs ou des entrepreneurs capitalisés (Cialdella et Navegantes-Alves, 2014 ; Gomes et al., 2015). Par ailleurs, la multiplication d'opérateurs industriels, exigeants en termes de régularité de volumes, pose question quant à l'inclusion des populations traditionnelles dans la filière, en même temps qu'elle offre une amélioration nette du niveau de vie.

La baie d'açaí étant très périssable, les possibilités de stockage sont limitées et coûteuses. Or le développement de plantations de terre ferme, en produisant des fruits de contre-saison, entre en concurrence directe avec les modes de production plus traditionnels, basés sur la gestion des terres inondables ${ }^{4}$.

D'un point de vue sanitaire, un stade critique de la filière est lié aux pratiques de cueillette ${ }^{5}$ et de transport, car les triatomes - insectes - porteurs de Trypanosoma cruzi, sont attirés par les paniers dans lesquels les fruits sont conditionnés (Aguiar, 2010). Dans l'Amazonie brésilienne, depuis 2006, des normes

4. Les plantations de terre ferme utilisent, en théorie, le cultivar BRS-Pará (Embrapa); la fertilisation chimique et l'irrigation font partie des itinéraires techniques. L'açaí natif des zones inondables recouvre une grande diversité génétique et bénéficie du mouvement des marées de l'estuaire amazonien, assurant irrigation et fertilisation naturelle.

5. Nous employons le mot cueillette car les techniques consistant à prélever les baies sur les arbres sont encore très proches des méthodes « traditionnelles », y compris dans les plantations. La cueillette est la première opération pouvant affecter la qualité du fruit. sanitaires et d'hygiène strictes ont été établies et sont appliquées sur les marchés externe et interne (Barbosa, 2010) après la démonstration de la transmission orale de la maladie de Chagas par la consommation d'açaí infecté. Il est donc impératif de tenir compte de ces aspects pour consolider l'ensemble des circuits de commercialisation longs et courts.

Nous avons donc cherché à approfondir les connaissances sur les circuits de commercialisation de la région métropolitaine de Belém, capitale du Pará, et la manière dont les transformateurs se réfèrent à la question de la qualité (sanitaire, gustative, esthétique). Notre hypothèse centrale est que la coexistence actuelle de circuits courts et globaux de commercialisation se base sur des attentes différentes quant à la qualité des pulpes, en lien avec les modes de consommation. Après avoir développé les cadres théoriques et les enjeux portant sur la standardisation des produits de la sociobiodiversité ${ }^{6}$ dans des contextes de globalisation des marchés, nous introduirons les résultats de l'étude en deux parties. Dans un premier temps, nous présenterons la diversité des circuits de commercialisation de la pulpe d'açaí qui dépasse la dichotomie local versus global et liée aux types de consommateurs et pratiques de consommation. Dans un second temps, nous établirons la relation entre ces différents circuits de consommation et les attributs de qualité auxquels se réfèrent les transformateurs. Notre discussion portera sur les enjeux de standardisation des produits et sur la formalisation des pratiques et savoirs locaux qui garantissent aujourd'hui la qualité pour les consommateurs locaux et une inclusion des populations traditionnelles dans la filière.

6. Biens et services générés à partir des ressources de la biodiversité locale et destinés aux chaînes productives d'intérêt pour les peuples et communautés traditionnelles et les agriculteurs familiaux (PCTAF) (PNSB, 2009). 


\section{L'açaí : une exception sur les marchés globalisés ?}

La valorisation économique des produits forestiers non ligneux ${ }^{7}$ est promue comme instrument de développement durable dans les régions forestières (Shackleton et al., 2007 ; Belcher et Schreckenberg, 2007). L'insertion des produits forestiers non ligneux dans les marchés de commodités est cependant controversée : exclusions d'acteurs, diminution de l'accès pour les populations modestes, surexploitations des ressources (Southgate, 1998; Méral et al., 2006 ; Ticktin, 2004). La tendance à substituer les pratiques de cueillette par des pratiques de domestication et de gestion est également critiquée : il ne faudrait alors plus parler de produits forestiers non ligneux mais d'agroforesterie ou d'agriculture (Leakey, 2012). À l'heure où Rostain (2016) montre que les forêts amazoniennes ont en partie été façonnées par l'homme, il semble vain d'établir une limite entre nature et culture concernant les produits forestiers non ligneux. Mais face aux changements en cours, la connaissance de la diversité des pratiques et des personnes qui vivent de la consommation et de la vente de ressources d'origine forestière permet de mieux comprendre les enjeux sous-jacents à la mise en avant de certaines pratiques plutôt que d'autres par différents types d'acteurs. L'appel de la rentabilité à court terme encourage parfois les populations traditionnelles à des pratiques favorisant le palmier açaí au détriment d'autres espèces. L'équilibre des écosystèmes ainsi que certaines fonctions alimentaires et médicinales s'en trouvent compromis (Araújo et Navegantes-Alves, 2015 ; Nogueira et al., 2005). Ce phénomène de domestication

7. Ensemble des productions intégrées du système forestier analysé. Elles ont une capacité de régénération totale par voie naturelle (FAO, http://www. fao.org/docrep/v0783f/v0783f02.htm, consulté le 14 septembre 2018). poussée à l'extrême est qualifié de processus d'«açaization» (Hiraoka, 1995). Parmi les populations traditionnelles, nous observons de fait un gradient de pratiques allant de l'extractivisme (où la seule intervention consiste à l'entretien du chemin d'accès aux palmiers) à la gestion d'espaces mono-espèce. Les nouveaux acteurs se caractérisent davantage comme "producteurs » plutôt que comme «extractivistes » et sont plus enclins à adopter les techniques de plantation de terre ferme mises en place par la recherche (Nogueira et al., 2005). Mais ils peuvent aussi recourir à des pratiques d'extraction dans les couverts forestiers leur appartenant -ou employer des tierces personnes à cette fin. Il n'existe donc pas, ou plus, de lien direct entre une «catégorie » de fournisseurs - populations traditionnelles versus agriculteurs-éleveurs - et un mode d'obtention du fruit (extraction versus culture). La différenciation se joue davantage sur des pratiques garantissant la saveur et le respect d'un mode de vie qui détermine le type de circuits de commercialisation dans lequel - ou lesquels - les cueilleurs sont insérés.

La garantie de cette différenciation, pour les consommateurs lointains, peut passer par la certification, qui, à l'exemple du commerce équitable, vise à construire des modalités de marché plus éthiques (Blanchet et Carimentrand, 2012). Sa mise en œuvre est difficile, notamment dans les pays du Sud (Walter et al., 2003 ; Boisvert et Caron, 2007). Par ailleurs, l'entrée des produits agricoles - ou produits forestiers non ligneux - sur les marchés des commodités, à travers l'adéquation aux normes d'exportation, tend à reléguer sur les marchés locaux des produits de qualité moindre (Estival, 2013). Les récents travaux portant sur la relocalisation des systèmes alimentaires amènent des contreexemples. Ils montrent que la connexion entre producteurs et consommateurs est 
la clé de réussite des circuits courts, qu'ils soient de niche ou alternatifs (Lamine et Chiffoleau, 2012; Lubello et al., 2017), par la construction de relations de proximité et de confiance (Schneider et Gazolla, 2017).

Dans le cas de l'açaí, malgré un essor vertigineux des circuits de commercialisation longs (vers d'autres États du Brésil et à l'international), la part de la consommation locale ne cesse d'augmenter, à travers un processus de valorisation gastronomique par les classes sociales aisées de la région (Diniz et Cerdan, 2017). Nous pourrions assister à une catégorisation des types d'açaí, même si actuellement la plupart des travaux économiques et de sciences de gestion analysent la «filière açaí » dans son ensemble, sans distinction de l'origine des fruits ou de la nature des produits consommés (Santos et al., 2014 ; Costa et al., 2018, Nogueira et al., 2013 ; Homma, 2006).

Nous avons dans un premier temps établi une typologie d'acteurs (cueilleurs, transformateurs) puis identifié les attributs de qualité mis en avant par chaque type d'acteurs. Enfin, nous avons établi des liens entre les circuits de commercialisation et les pratiques de cueillette et de production, de transport des fruits jusqu'aux lieux de transformation.

Une recherche exploratoire a été conduite entre les mois de juillet et septembre 2016 auprès de six transformateurs considérés comme «locaux », de renom dans la ville (I1, I2, L4 cf. tableau 1, en annexe). Puis nous avons enquêté auprès de quatre industriels qui communiquent autour de leur démarche de qualité (sites internet) ainsi que par recommandation (G1, tableau 1). Dans chacun des établissements sélectionnés, une ou plusieurs personnes ont été rencontrées en fonction de leur position (propriétaire, gérant, responsable des ventes, des achats, du marketing, de la qualité) afin d'obtenir les informations souhaitées sur les stratégies de vente (types de consommateurs, lieux), la qualité (caractérisation, contractualisation et tarification différentiée éventuelle), les stratégies d'achat (types de cueilleurs, lieux, organisation de la cueillette et du transport).

Deux marchés spécialisés dans l'açaí (Ver-o-peso et Icoaraci) ont également fait l'objet d'enquêtes compréhensives. Dans le temps limité de l'étude, il s'est avéré difficile de multiplier les entretiens avec les industriels et les commerçants.

\section{Les goûts au cœur de la coexistence de circuits diversifiés}

Dans les villes amazoniennes et à Belém en particulier, les consommateurs opposent fréquemment l'açaí local, basé sur des circuits courts, produit dans les règles de l'art et savoureux, à l'açaí industriel, pasteurisé et congelé, destiné à l'exportation. Nos résultats montrent que si ces discours sont en partie fondés, ils masquent un panorama bien plus diversifié des circuits de commercialisation qui propagent l'actuelle «fièvre de l'açaí ». La manière dont les différentes entreprises, qu'elles soient artisanales ou industrielles, s'expriment sur la question de la qualité dépend de leurs profils de leurs stratégies commerciales et du type de consommateur visé.

\section{Les transformateurs " industriels ॥ : à la recherche de spécification}

Le premier résultat de l'étude a concerné les quatre entreprises industrielles tournées vers l'exportation. Par des mécanismes de certification ou des procédés différenciés de transformation, trois d'entre elles cherchent à anticiper l'installation d'industries concurrentes de grande taille, originaires du Sud du pays. Ces transformateurs exportent vers les États du Sud, Centre-Sud, Nord-Est du Brésil et vers l'international (figure 1), de la pulpe dite 


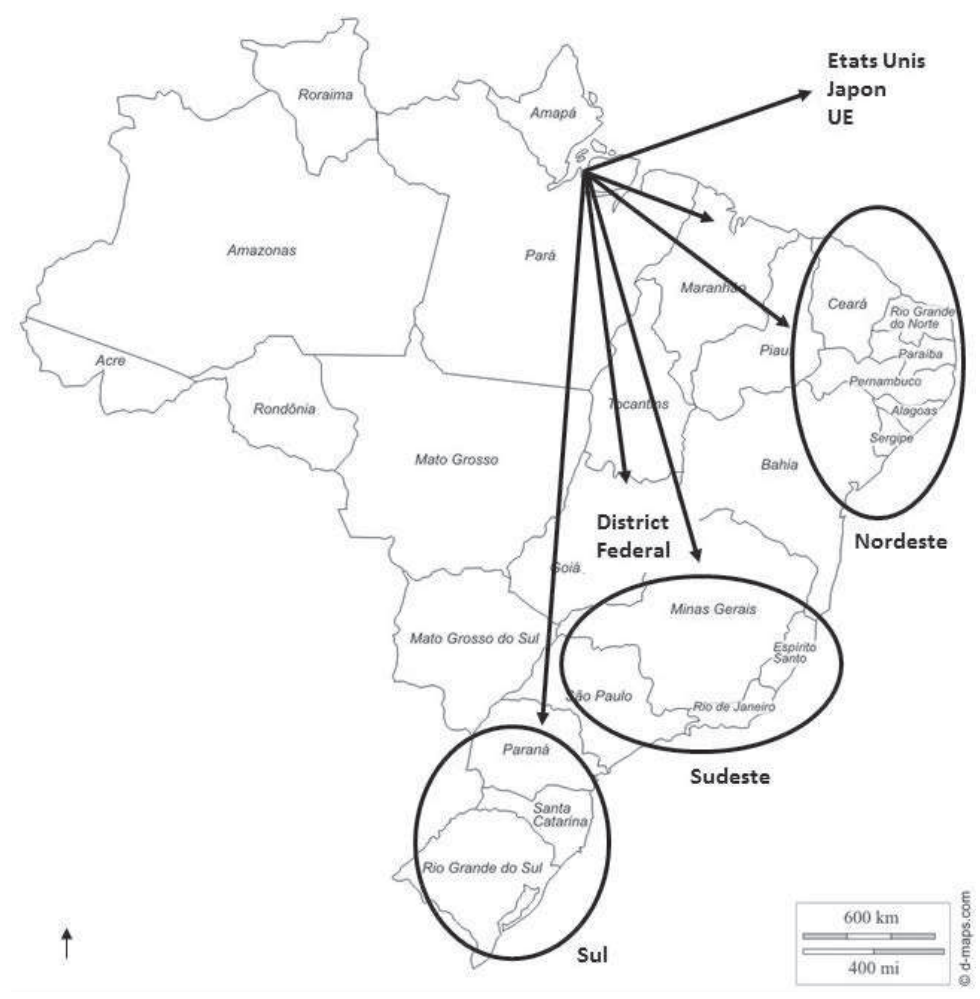

Source : enquêtes de terrain (fond Google Maps).

«populaire» (contenant de 8 à $11 \%$ de matière sèche, Nogueira et al., 1995). Cette pulpe, pasteurisée et congelée, entre la plupart du temps comme matière première pour des mélanges, glacés ou non. Une seule de ces entreprises (B1) de taille moyenne (46 employés) et tenant pour origine une structure artisanale familiale achemine principalement de la pulpe moyenne (de 11 à $14 \%$ de matière sèche) dans les États du Nord-Est du Brésil. Les consommateurs de ces États consomment préférentiellement la pulpe pure et plus épaisse, à la manière amazonienne.

Parmi les quatre industries enquêtées, trois ont obtenu une certification biologique et deux d'entre elles possèdent une certification de commerce équitable. Les entreprises justifient cette démarche pour garantir aux consommateurs distants le respect de la population et de la biodiversité amazonienne. Elles comptent ainsi affirmer un différentiel vis-à-vis de grands groupes industriels venant du Sud du pays et cherchant à s'implanter en Amazonie. Pour ces derniers, il s'agit de réduire les coûts en s'affranchissant des petites entreprises qui leur fournissaient la matière première (pulpe d'açaí pure). Jusqu'à présent ces implantations se soldent par des échecs du fait de l'atomisation des acteurs de la filière (figure 2) et de l'importance des relations de gré à gré, basées sur la confiance (ou la dépendance, comme le relate Pegler [2011]). Ainsi, la certification, qui marque un passage du contrat de confiance au 
contrat formel avec les cueilleurs et les intermédiaires, garantit une différenciation au regard d'une compétitivité qui risquerait de s'accroître dans le futur avec l'installation d'industries de grande taille.

Cette inquiétude est partagée par une entreprise employant jusqu'à 100 personnes, G2 (tableau 1, annexe), qui s'engage sur la voie de la santé en projetant de lyophiliser l'açaí. Transformer la pulpe en poudre lui permet de se positionner sur les marchés européens et de réduire les frais de transport en s'affranchissant des chambres frigorifiques et du poids de l'eau contenue dans les pulpes.

Pour ces entreprises, la régularité des volumes vendus est primordiale. Or les fruits d'açaí sont très périssables et le stockage à froid extrêmement coûteux en Amazonie. Le palmier Euterpe oleracea Mart. a la particularité d'être très sensible aux régimes des pluies. Ainsi, la période de cueillette des fruits d'açaí s'étale sur l'ensemble de l'année, du nord au sud de l'estuaire amazonien : de décembre à avril et en juin-juillet près de Belém; d'août à novembre/décembre vers le sud, à la frontière du Maranhão; de mars à juillet en Amapá (figure 2). Par la diffusion de pratiques intensives, la région principale de production de l'açaí, le Baixo Tocantins, produit des fruits sur la quasi-totalité de l'année. Ainsi, les industries d'exportation s'approvisionnent donc sur un rayon de plusieurs centaines de kilomètres du site de transformation (figures 2 et 5 ).

\section{Les intermédiaires : l'açaí comme ancrage local d'une autre activité}

Un second résultat est l'identification de deux transformateurs que nous qualifions d'intermédiaires, par leur taille comparable à celle des industries (tableau 1, annexe) et par leur fonctionnement constant sur l'année. Ces établissements (un glacier et un restaurant), anciens de plusieurs générations, doivent leur renommée à leur ancrage local et à la qualité de leurs produits, dont l'açaí, vendu principalement en concentration moyenne (entre 11 et $14 \%$

Figure 2. Lieux d'approvisionnement des industriels et intermédiaires

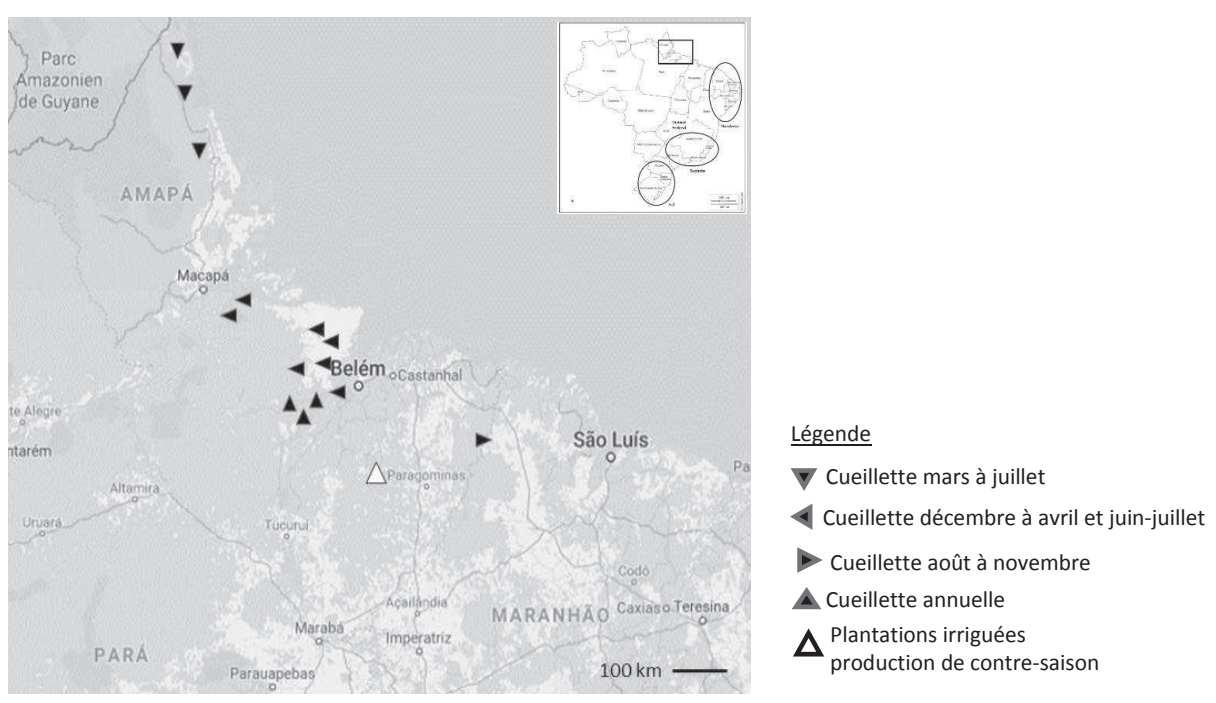

Source : enquêtes de terrain (fond Google Maps). 
de matière sèche). Leurs marchés se situent dans la ville ou l'agglomération de Belém. Ces deux transformateurs sont touristiques et mettent en avant l'açaí comme « image de marque ». Afin d'assurer la continuité de l'offre, ces transformateurs sont amenés à stocker de la pulpe congelée mixée ou de s'approvisionner en fruits dans des régions éloignées en dehors des périodes de cueillette de la région de Belém (figure 2). Dans les deux cas, cette pratique affecterait la saveur du fruit. Il est intéressant de noter que ces établissements ont gagné la confiance des consommateurs locaux et conservent une image de qualité, alors qu'ils sont moins regardants sur la saveur que les transformateurs artisanaux. Les produits vendus, qui sont les produits dérivés sucrés et parfois de l'açaí de terre ferme (supposé de saveur différente que l'açaí des zones inondables), sont acceptés par la population urbaine de Belém et des touristes. Ces produits ne trouvent pas de débouché localement lorsqu'ils sont proposés par les industriels (BE2, G2, BL1), faute d'acceptation par les consommateurs.

Cette confiance est en partie due à l'ancienneté des établissements, mais également aux modes de transformation de la pulpe : transformation sur place et à la demande pour le restaurant, sous-traitance avec plusieurs transformateurs artisanaux de Belém pour le glacier. Les intermédiaires sont également marqueurs de changement dans les habitudes de consommation alimentaire qui touche l'ensemble de la région en milieu urbain et rural, vers des aliments plus riches et plus sucrés.

\section{Les circuits courts de niche, vers la " gourmétisation "}

Les transformateurs artisanaux de l'agglomération de Belém sont au nombre approximatif de 3000 et fournissaient localement près de 470000 litres de pulpe en $2012^{8}$. Ces chiffres varient en fonction des

8. Source : Secrétariat de l'agriculture, SAGRI. saisons et des années ; de nombreux points de vente sont des microstructures qui ouvrent uniquement pendant la période de cueillette de la région de Belém (décembre à mars/avril). En 2007, des travaux établissent une corrélation entre la contamination de l'açaí par le Tripanosome cruzi et des déclarations de maladie de Chagas chez l'être humain (Aguiar, 2010). Les transformateurs artisanaux, menacés de fermeture, s'organisent et obtiennent que la pasteurisation soit facultative pour la consommation de pulpe fraîche au niveau local (Bezerra, 2009). Les résultats de nos enquêtes montrent que certains transformateurs artisanaux sont aujourd'hui dans une démarche avancée de recherche de qualité, non plus seulement sanitaire, mais également gustative. Pour cela, ils sélectionnent leurs fournisseurs en fonction de leurs pratiques de culture, de cueillette et de transport, comme nous le verrons dans la partie suivante, ainsi qu'en fonction de leur proximité à la ville (figure 3). Ils vendent principalement de la pulpe à concentration élevée en matière sèche (açaí spécial, de plus de $14 \%$ de MS, tableau 1, annexe). Les transformateurs artisanaux encouragent donc le développement de la cueillette et de la production d'açaí dans les îles. Ils contribuent ainsi à améliorer le tissu socio-économique local.

\section{Des goûts liés à l'origine : des pratiques et des lieux}

La préoccupation première des transformateurs interrogés, en termes de qualité, porte sur les aspects sanitaires. Tous, sans exception, appliquent le code des bonnes pratiques de transformation (tableau 2 en annexe).

Au-delà de la qualité sanitaire, les transformateurs s'accordent également sur les principaux critères de qualité de l'açaí: couleur, saveur, odeur, rendement ( $t a-$ bleau 2). L'origine n'a été explicitement 


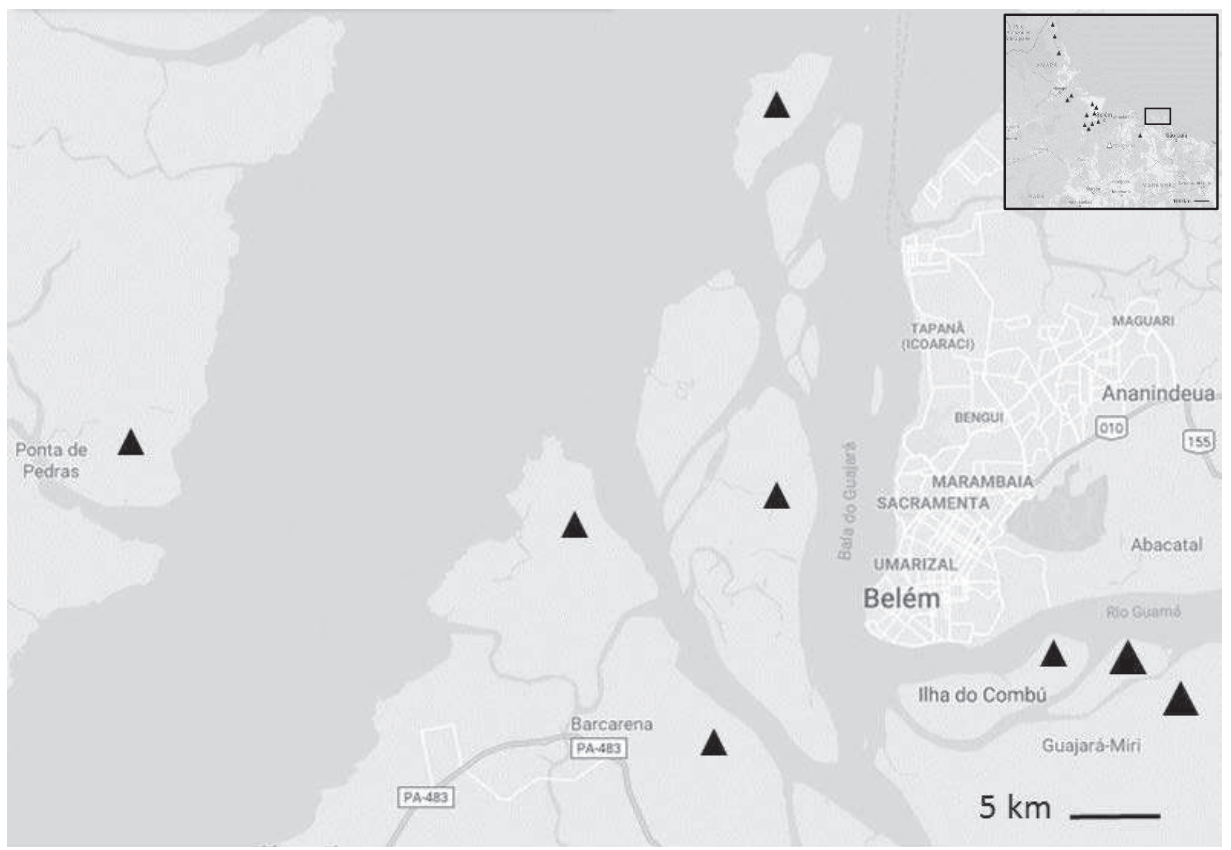

Note : Le nombre de triangles indique les localités et non le nombre de fournisseurs, impossible à établir. Les échanges sont nombreux et quotidiens entre les cueilleurs et les transformateurs de Belém.

Source : enquêtes de terrain (fond Google Maps).

citée qu'une fois; elle est cependant centrale et liée à tous les autres critères. Implicitement, les transformateurs y font référence tant sur le plan de la localisation géographique (zone de plantation de terre ferme versus zone inondable), de distance (temps post-cueillette) que sur les pratiques de production, cueillette et transport, mises en œuvre tout au long des canaux de distribution. De fait, la connaissance approfondie de ces pratiques par les transformateurs est un gage de qualité gustative.

Les critères de goût et d'odeur sont également liés; les transformateurs industriels et intermédiaires s'y réfèrent le plus souvent vis-à-vis du processus de fermentation. En effet, un açaí fermenté aura une odeur et un goût acide caractéristique.

La couleur est le critère "marketing » de l'açaí. Toutes les personnes interrogées considèrent qu'un bon açaí possède une couleur violet foncé, étant la combinaison du lieu d'origine (violet en zone inondable et marron en terre ferme), de la maturité du fruit et de la qualité du transport. Les régions d'Igarapé-Miri et Cametá, situées dans le principal bassin de production (le Baixo Tocantins), ont été citées à plusieurs reprises comme fournissant un açaí aux tons gris-cendré, moins prisé localement.

Afin d'obtenir une coloration équilibrée et acceptable tout au long de l'année par les consommateurs, les industriels mélangent les fruits de plusieurs origines, employant le terme anglais de blend comme signe d'un alignement aux normes du commerce international (BE2, G2). Dans la plupart des cas, la pulpe d'açaí ne constitue qu'un ingrédient parmi d'autres dans le produit final ; des modifications ponctuelles de la 
coloration de la pulpe sont encore acceptées par les clients. Les consommateurs locaux - et du Nordeste - se refusent à consommer de l'açaí marron de terre ferme. Ainsi l'entreprise BL1 se positionne essentiellement sur le marché nordestin et a construit sa réputation sur les savoir-faire d'un transformateur artisanal. Il stocke la pulpe congelée provenant des îles plusieurs mois durant au lieu de s'approvionner dans les terres fermes.

La saveur est le deuxième critère le plus cité. Il a été cependant très difficile de détailler ce qui distingue un bon açaí d'un médiocre en termes de saveur. Dans la plupart des cas, la saveur correspond à la fraîcheur des fruits et de la pulpe et s'oppose au goût de fermentation. Un ensemble de pratiques, garantissant la fraîcheur, est exigé par les transformateurs artisanaux (L2, L3, L4, tableau 2). Il s'agit de minimiser le temps post-cueillette et de soigner le conditionnement des fruits pendant le transport, de manière à éviter le contact avec l'eau. Étant donné que la plupart des fruits sont acheminés en petits bateaux, ce dernier point constitue un véritable challenge pour les transporteurs.

L'odeur est très liée à la saveur, mais ce critère n'a été cité que trois fois (I1, L1, L4), sans doute parce qu'elle n'est perceptible par le consommateur lambda qu'à partir d'un état de fermentation avancé de la pulpe.

Le rendement est un critère important, cité explicitement par deux industries (BE2, BL1) et un transformateur artisanal (L1). Ce critère a été implicitement abordé par les autres transformateurs car le rendement n'est pas perçu comme un critère lié à la qualité mais à la rentabilité. Seule G2, qui travaille avec de grands volumes de fruits tout au long de l'année, n'a pas cité le rendement pulpe/fruit comme une préoccupation au cours de l'entretien. Pour ce critère, l'origine est mise en avant, en distinguant, une nouvelle fois, l'açaí des îles et des zones inondables comme étant d'un meilleur rendement en pulpe que l'açaí de terre ferme considéré plus sec.

Notons que, pour les industries, la qualité passe par des mécanismes de certification formels : biologique et équitable. Or, dans la grande majorité des cas, la production et la cueillette sont encore le fait de populations traditionnelles (ribeirinhos) travaillant manuellement et sans intrants chimiques, donc a fortiori dans des systèmes de production biologique. Certifications coûteuses, mais faciles à obtenir, elles fournissent au consommateur lointain l'image d'un produit préservant la nature et les populations traditionnelles. Ces certifications ne considèrent pas les critères de goût et esthétiques exigés localement par les consommateurs. La prise en compte de ces exigences conduit à des arrangements informels garantissant une qualité " gourmet », se basant sur le respect d'un ensemble de pratiques essentiellement connues et exclusivement citées par les transformateurs artisanaux. Parmi ces pratiques, trois sont en lien avec les normes sanitaires en vigueur (pratiques 1, 4, 5, tableau 3 en annexe).

Un moyen utilisé pour ralentir la fermentation des fruits est de transporter les paniers au-dessus de blocs de glace placés en fond de cale des bateaux. Mais cette pratique a tendance à humidifier les fruits (pratique 6, tableau 3 en annexe) et diminuer leur qualité. Le temps de transport et l'humidité des fruits sont alors liés : plus les fruits arrivent de loin, plus les chances qu'ils arrivent «mouillés » augmentent. Ce qui explique que les transformateurs et commerçants locaux exigent que les fruits voyagent le moins longtemps possible (une demi-journée).

Les pratiques de cueillette citées comme ayant une influence sur la saveur des fruits correspondent au soin fourni lors de la manipulation des fruits (pratique 2, ne pas griffer les fruits, cité par L4; et cueillir les 
fruits à maturité, cité par BE2, G2, L2, L3, L4). Il est intéressant de noter que la pratique est avant tout citée par les transformateurs artisanaux qui entretiennent davantage de proximité avec les cueilleurs. Dans le cas de l'entreprise BE2, la pratique a été citée par l'ingénieur agronome en charge de la contractualisation avec les cueilleurs, qui développe donc des relations directes avec ces derniers. La maturité s'obtient lorsque $90 \%$ des fruits se couvrent d'une fine pellicule blanche en surface.

\section{Création de proximité entre cueilleurs, transporteurs et transformateurs}

Les transformateurs artisanaux se fournissent en fruits provenant des îles proches de la ville de Belém, de manière à garantir le plus de fraîcheur possible : les distances sont le plus souvent inférieures à $10 \mathrm{~km}$, parfois $45 \mathrm{~km}$ (correspondant à deux heures de barque). Nous avons identifié trois types d'approvisionnement (figure 4). Dans le premier cas (L1, L3, L4), le transformateur est également fournisseur (il emploie des cueilleurs sur ses propres terres). Il organise lui-même le transport ou bien rémunère un transporteur professionnel (en bateau de l'île au port, puis en camion jusqu'à l'atelier de transformation). Dans ce type d'approvisionnement, il n'y a pas d'achat de matière première. Le transformateur utilise sa propre production; la cueillette est souvent réalisée par des travailleurs métayers. Ce système permet par ailleurs un contrôle optimum des pratiques de cueillette visant la qualité du produit. Dans un second cas, le cueilleur fournit la matière première au transformateur, qui assure lui-même le transport des fruits. La négociation peut se faire au débarquement de la marchandise ou au préalable, par un accord oral établi pour les volumes et les prix sur l'ensemble de la saison ou au coup par coup, par téléphone. Dans un dernier cas, le cueilleur vend ses fruits à des intermédiaires qui négocient les volumes et les prix, sur les marchés ou au préalable avec les transformateurs.

À l'inverse, les transformateurs industriels doivent composer avec des distances géographiques et relationnelles plus grandes (figure 5). La plupart des industries se situant en dehors de la ville, les distances du port à la fabrique sont de l'ordre d'une centaine de kilomètres. La stratégie visant à s'approvisionner le plus longtemps possible dans l'année, de manière à limiter les stocks de pulpe congelée, oblige les industries à s'approvisionner loin : entre 125 et $145 \mathrm{~km}$ en bateau, pour l'açaí des îles et de zones inondables,

Figure 4. Types d'acheminement des fruits des cueilleurs aux transformateurs artisanaux et aux consommateurs

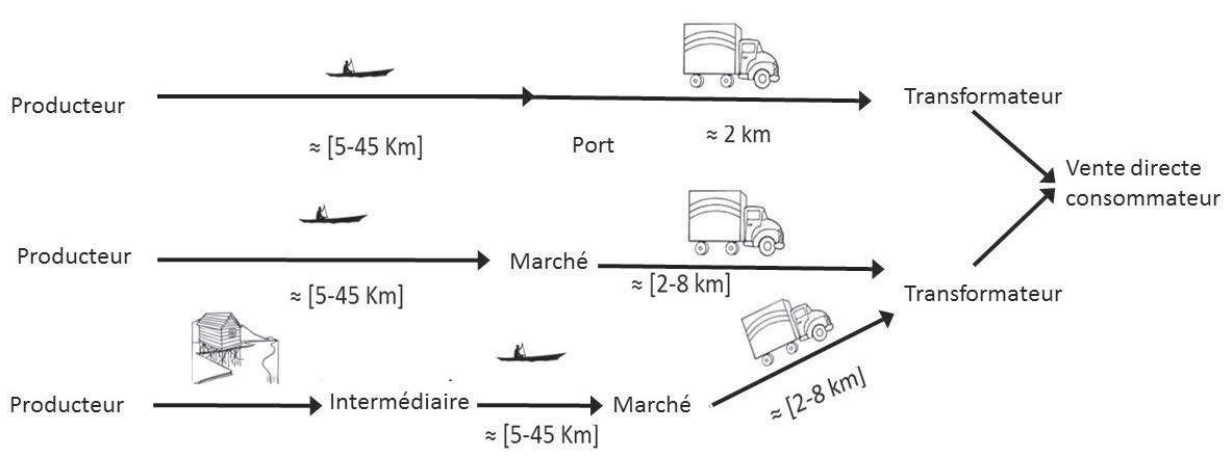




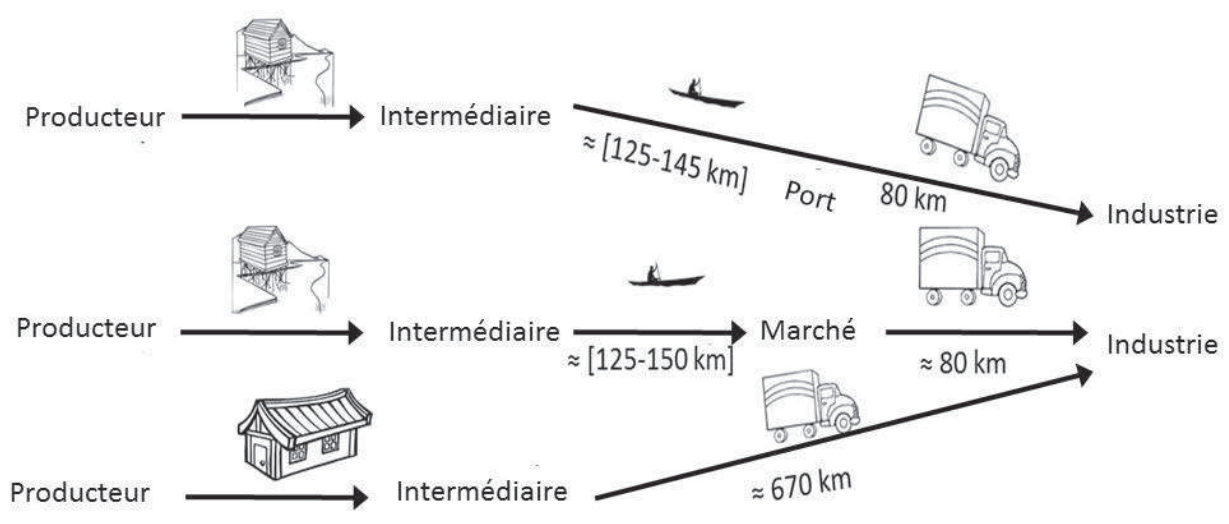

Source : recherche de terrain.

plus de $700 \mathrm{~km}$ pour l'açaí provenant d'Amapá ou de terre ferme en provenance du Maranhão (figure 2). Dans ce cas, il n'y a aucun contact entre l'industrie et les cueilleurs : l'intermédiaire est le seul à négocier les prix et la qualité. Il en va de même lorsque l'açaí vient des îles éloignées et où la négociation se réalise sur les marchés, au moment du débarquement des fruits. Dans ce cas, la traçabilité des fruits est difficile à établir, voire impossible. Les industries engagées dans des démarches de certification biologique et équitables recherchent néanmoins à recréer une certaine forme de proximité avec les cueilleurs. Celle-ci s'organise en établissant un contrat d'engagement via la figure de l'intermédiaire. Ce dernier devient garant des bonnes pratiques de production, de cueillette et de transport vis-à-vis de l'entreprise en même temps qu'il garantit des prix supérieurs au marché pour les cueilleurs dont les pratiques respectent les fruits et l'environnement (labels bio) et le respect d'un mode de vie (labels équitables). L'intermédiaire devient alors médiateur et formateur auprès des communautés de cueilleurs. L'industrie BE2 compte ainsi sur 10 intermédiaires-médiateurs, qui accompagnent chacun 50 cueilleurs. À partir des transformateurs, la destination des pulpes est distante (plusieurs centaines à des milliers de kilomètres) et variable : nouveaux grossistes, entreprises agroalimentaires, points de vente au détail. Cette partie de la chaîne de distribution n'a pas été étudiée en détail.

Dans ces nouvelles configurations d'échanges, nous assistons à une modification du rôle des marchés localisés sur les débarcadères des fruits d'açaí. Le plus important et le plus connu est le marché du Ver-o-peso. Au plus fort de la période de cueillette, le marché s'anime plusieurs fois par jour en fonction des lieux d'origine des fruits, ainsi que des marées (l'açaí pouvant venir d'Amapá, à plusieurs jours de bateau). À l'intersaison - pour la région de Belém -, le marché du Ver-o-peso fonctionne toujours, avec cette fois l'açaí provenant de terre ferme, de l'État du Maranhão... les intermédiaires et cueilleurs savent donc que l'açaí débarqué au Ver-o-peso trouvera toujours acheteur, quelle qu'en soit la qualité. Si certains transformateurs organisent toujours les transactions et le débarquement des fruits au marché du Ver-o-peso, la plupart des négociations sont conclues auparavant par téléphone. Le Ver-o-peso est aujourd'hui 
RECHERCHE Nathalie CIALDELLA, Edfranklin SILVA, Livia NAVEGANTES-ALVES, Janaina DEANE DE ABREU SÁ DINIZ

délaissé par les acteurs engagés dans les circuits courts et marchés de niche « gourmet » au profit de certains marchés secondaires et moins fréquentés (port da Palha, de Icoaraci, de Guama, etc.).

\section{Garantir le goût, un enjeu pour les circuits courts}

Les résultats montrent que la dichotomie entre transformation artisanale-locale versus industrielle-d'exportation est bien plus diverse et poreuse, tout d'abord sur le plan de l'approvisionnement en fruits. L'açaí de terre ferme et « endormi », ayant parcouru des centaines de kilomètres sur des blocs de glace en fond de cale, n'est pas réservé aux industries, mais peut également fournir les transformateurs « locaux » tels les intermédiaires. Inversement, les populations traditionnelles participent à tous les types de circuits de commercialisation. Qui plus est, le fait que les prix sont élevés quels que soient les circuits de commercialisation laisse une certaine flexibilité aux cueilleurs et aux intermédiaires. Les industriels, moins exigeants en termes de qualité gustative, garantissent aux cueilleurs un débouché pour les invendus des circuits locaux. La coexistence de différents circuits de commercialisation au sein des territoires n'est donc pas toujours concurrentielle; les connexions entre circuits peuvent être motrices de développement territorial, comme le montrent Schneider et al. (2016) au sud du Brésil par l'analyse des marchés imbriqués (Ploeg et al., 2012). Dans le présent article, nous avons principalement abordé les circuits courts de marché de niche. Il s'agirait de compléter les connaissances sur les circuits courts qui garantissent l'accès et la qualité du produit à des consommateurs modestes. Les travaux de Sousa et al. (2018) montrent que d'autres marchés se maintiennent pour des produits rendus invisibles par la « fièvre » de l'açaí, en particulier le palmier miriti (Mauritia flexuosa) dans les villes périphériques de Belém.
Que ce soit dans les circuits locaux ou d'exportation, la question de l'engagement et de la fidélisation du cueilleur devient primordiale ; la différenciation de la production, sur la base d'un bonus payé à la qualité entre alors en jeu. Cette qualité peut être basée sur des critères standardisés à l'international (agriculture biologique et commerce équitable) garantissant au consommateur étranger la préservation de la forêt et des populations amazoniennes. Nous assistons ainsi à l'émergence de contrats formels entre industries et cueilleurs, non sans difficultés, mais qui tend à imposer les standards de qualité associés. Ces dynamiques de certification internationales en sont à leurs prémisses et demandent à être accompagnées et évaluées, car elles ont dans d'autres contextes montré des dynamiques d'exclusion et d'accroissement des inégalités (Carimentrand, 2011 ; Belleti et al., 2016).

À l'inverse, au niveau local, la garantie de la qualité se base sur des mécanismes informels qui valorisent le goût et le mode de consommation traditionnel, pur, associé à la mise en valeur de modes de productions souples et séculaires pratiqués dans les îles. Or c'est une qualité non explicite, s'appuyant davantage sur un consensus collectif, un ensemble de relations sociales de proximité et de confiance construites parfois sur plusieurs générations. Lors des entretiens, les relations de confiance, d'interconnaissance et de respect mutuel ont uniquement été citées. Les relations de dépendance, encore très communes entre les cueilleurs métayers et les propriétaires fonciers ont été relatées dans l'État d'Amazonas (Pegler, 2011), mais n'ont pas été identifiées dans nos enquêtes. En partie car les entretiens ont été conduits avec les transformateurs, acteurs ayant une position de force dans la gouvernance des filières. À l'instar d'autres produits de la sociobiodiversité ou de nombreux produits alimentaires protégés par des indications géographiques, la préservation de cet équilibre 
pourrait passer par une meilleure définition de ce que les consommateurs, transformateurs et cueilleurs d'Amazonie entendent par « saveur » afin de préserver un système alimentaire diversifié et ancré dans le territoire (Cruz et Schneider, 2010). La mise en place d'un système de garantie participative (Lemeilleur et Allaire, 2018), développé notamment dans le Sud du Brésil pour les produits agroécologiques, semble la voie la plus appropriée pour préserver la diversité des savoir-faire et savoir consommer l'açaí comme un bien commun amazonien.

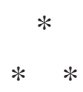

La croissance du marché de l'açaí porté par la transformation industrielle a entraîné de profondes modifications dans les modes de consommation et de commercialisation du fruit. Notre étude montre que ces transformations sont multidimensionnelles : nouveaux produits de consommation obtenus à partir de pulpe dont on attend peu de la qualité gustative, nouveaux acteurs tels que les cueilleurs de terre ferme. À l'opposé, l'émergence de l'açaí hors des frontières amazoniennes semble avoir réveillé localement des démarches de qualité et de renforcement des proximités.

Ainsi, jusqu'à présent, quatre types de circuits de commercialisation, liés aux pratiques, aux lieux de consommation (pulpe versus mix; local versus global) et à la certification (générique versus certifié) existent dans la région. Ceci permet la coexistence de pratiques diversifiées, de la gestion et l'aménagement en zone inondable à la plantation de terre ferme. Jusqu'à présent, les standards de consommation locale assurent un approvisionnement en pulpe de qualité sur place, ce qui différencie l'açaí d'autres produits tels le cacao, le café, pour lesquels la meilleure qualité est destinée à l'exportation. L'açaí représente en ce sens un cas de marché de commodités inversé. Mais le poids des consommateurs extérieurs à la région, qui ne cesse d'augmenter, pourrait bien in fine imposer ses standards de "goût » et remettre en cause la coexistence actuelle des circuits de commercialisation, en particulier l'inclusion des populations traditionnelles dans la filière et l'accès à des produits de qualité pour les consommateurs locaux. D'autres produits de la sociobiodiversité ont connu des tensions similaires et des dynamiques de «patrimonialisation », tels le guaraná (Paullinia cupana) et le quinoa (Chenopodium quinoa). Ces exemples montrent l'importance $\mathrm{du}$ processus de construction sociale autour de la reconnaissance des populations traditionnelles, détenant les savoirs associés à ces produits. Il est donc primordial d'approfondir les connaissances sur les changements en cours dans les différents maillons de la filière, de mieux connaître les mécanismes informels construits pour les circuits courts afin de définir, notamment, des critères pertinents localement pour l'évaluation de la qualité.

Cette recherche a bénéficié du soutien du programme Guyamazon, dans le cadre du projet Frontagui. 


\section{RÉFÉRENCES BIBLIOGRAPHIQUES}

Aguiar F. S. (2010). Avaliação da fermentação espontânea dos frutos de Euterpe Oleracea durante o período pós-colheita e suas possíveis implicações sobre atração de triatomínios. Belém, Universidade Federal do Pará, Dissertação de Mestrado, 86 p.

Aguiar F. S., Rogez H., Menezes V. R. (2013). Spontaneous fermentation of Euterpe oleracea fruits during the postharvest. Postharvest Biology and Technology, ${ }^{\circ}$ 86, pp. 294-299.

Araújo C. T. D., Navegantes-Alves L. F. (2015). Do extrativismo ao cultivo intensivo do açaizeiro (Euterpe oleracea Mart.) no estuário amazônico: sistemas de manejo e suas implicações sobre a diversidade de espécies arbóreas, Revista Brasilira de Agroecologia, vol. 1, n 10, pp. 12-23.

Azevedo J. R., Kato O. R. (2007). Sistema de manejo de açaizais nativos praticados por ribeirinhos das ilhas de Paquetá e Ilha Grande, Belém, Pará, in Agricultura familiar, políticas públicas e inclusão social: anais. Fortaleza, Embrapa Agroindústria Tropical.

Barbosa R. L. (2010). Transmissão oral do trypanosoma cruzi pela polpa de açaí em camundongos. Dissertação (Mestrado em Parasitologia), Instituto de Biologia da Universidade Estadual de Campinas, Campinas/SP, $97 \mathrm{p}$.

Belcher B., reckenberg K. (2007), Commercialisation of Non-timber Forest Products: A Reality Check. Development Policy Review, $\mathrm{n}^{\circ} 25$, pp. 355-377. doi:10.1111/j.14677679.2007.00374.x

Belleti G., Chabrol D., Spinsanti G. (2016). Échapper au piège de la «qualitéexclusion » dans les indications géographiques : réflexions sur le cas du poivre de Penja. Cahiers Agricultures, n ${ }^{\circ} 25,55002$. doi:10.1051/cagri/2016034

Béreau D. (2001). Huiles et fractions insaponifiables de huit espèces de palmiers amazoniens. Thèse, INP, Toulouse.

Bezerra V. S. (2009). As boas práticas de fabricação na amassadeira de açaí. Embrapa Amapá, 124 p.
Blanchet V., Carimentrand A. (2012). Dictionnaire du commerce équitable. Versailles, Quæ. doi:10.3917/quae.blanc.2012.01

Boisvert V., Caron A. (2007). Valorisation économique des ressources et nouveaux marchés. In Aubertin C., Pinton F., Boisvert V., les marchés de la biodiversité, Paris, IRD, pp. 195-218.

Brondizio E. (2008). Amazonian Caboclo and the açai Palm. Forest Farmers in the Global Market. New York, Ed. Botanical Garden Pr Dept., 403 p.

Brondízio E. S., Safar C. A., Siqueira A. D. (2002). The urban market of Açaí fruit (Euterpe oleracea Mart.) and rural land use change: Ethnographic insights into the role of price and land tenure constraining agricultural choices in the Amazon estuary. Urban Ecosystems, ${ }^{\circ}$ 6, pp. 67-97

Carimentrand A. (2011) Les commerces équitables du quinoa: une analyse de la diversité des filières. Canadian Journal of Development Studies, vol. 3, $\mathrm{n}^{\circ} 32$, pp. 313-323, halshs-00771554.

Costa F. A., Fernandes D. A., Sousa C. N. (2018) Constituição, Situação e Dinâmica de Arranjos Produtivos Locais: O caso do APL de Açaí na Região do Grão-Pará (2003 a 2010). Análise Econômica (UFRGS), vol. 36, pp. 109-137.

Cialdella N., Navegantes-Alves L. (2014). La ruée vers l'açaí (Euterpe oleracea Mart.) : trajectoires d'un fruit emblématique d'Amazonie. Tiers Monde, $\mathrm{n}^{\circ} 220$, pp. 119-135.

Cruz F. T., Schneider S. (2010). Qualidade dos alimentos, escalas de produção e valorização de produtos tradicionais, Revista Brasilira de Agroecologia, vol. 2, $\mathrm{n}^{\circ} 25$, pp. 22-38.

Diniz J. D. A. S., Cerdan, C. (2017). Produtos da sociobiodiversidade e cadeias curtas: aproximação socioespacial para uma valorização cultural e econômica. In Gazolla M., Schneider S. (Org.), Cadeias curtas e redes agroalimentares alternativas: negócios $e$ mercados da agricultura familiar, Porto Alegre, Editora UFRGS, pp. 259-280. 
Estival K. G. S. (2013). Construção social do mercado de qualidade do cacau no Brasil. Thèse, Université Fédérale Rurale de Rio de Janeiro (UFRRJ).

Gomes M. R., Padilha de Araujo F. D., Gomes M. O., Resque A. G. L., Cialdella N. (2015). Emergência do plantio de açaí: rumo inverso a agroecologia? O caso de Paragominas. Cadernos de Agroecologia, vol. $10, \mathrm{n}^{\circ} 3,5 \mathrm{p}$.

Hiraoka M. (1995). Land-use changes in the Amazon Estuary. Global Environmental Change: Human and Policy Dimensions, vol. 5, n 4, pp. 323-336.

Homma A. K. O., Nogueira O. L., Menezes A. J. E. A., Carvalho J. E. U., Nicoli C. M. L., Matos G. B. (2006). Açaí. Novos desafios e tendências. Ciências \& Desenvolvimento, vol. $2, \mathrm{n}^{\circ} 1$, pp. 78-23.

Lamine C., Chiffoleau Y. (2012). Reconnecter agriculture et alimentation dans les territoires dynamiques et défis. Pour, n ${ }^{\circ} 215-216$, pp. 85-92.

Leakey R. R. B. (2012). Non-timber forest products - a misnomer? Journal of Tropical Forest Science, n 24, pp. 145-146.

Lemeilleur S., Allaire G. (2018). Système participatif de garantie dans les labels du mouvement de l'agriculture biologique. Une réappropriation des communs intellectuels. Économie rurale, $\mathrm{n}^{\circ} 365$, pp. 7-27.

Lubello P., Falque A., Temri L. (2017). Systèmes agroalimentaires en transition. Versailles, Quæ.

Menezes E., Deliza R., Chan H. L., Guinard J.-X. (2011). Preferences and attitudes towards açaí-based products among North American consumers. Food Research International, vol. 7, n 44, pp. 1997-2008.

Méral P., Raharinirina V., Andriamahefazafy F., Andrianambinina D. (2006). La valorisation économique des forêts: entre filière et territoire. Économie rurale, n ${ }^{\circ} 294-295$, pp. 74-89.

Nogueira O. L., Carvalho C. J. R., Muller C. H., Galvão E. U. P., Silva H. M. E., Rodrigues J. E. L. F., Oliveira M. S. P., Carvalho J. E. U., Rocha Neto O. G., Nascimento W. M. O., Calzavara B. B. G. (1995). A cultura do açaí. Brasília, EMBRAPA, 49 p.
Nogueira O.L., Figueiredo F. J.C., Müller A.A. (2005). Açai, sistemas de produção. Embrapa, en ligne : https://www.embrapa.br/ busca-de-publicacoes/-/publicacao/408196/ acai.

Nogueira A. K. M., Santana A. C., Garcia W. S. (2013). A dinâmica do mercado açaí fruto no estado do Pará: de 1994 a 2009. Ceres, vol. $3, \mathrm{n}^{\circ} 60$, pp. 324-331.

Pegler L. (2009). Cadeias Produtivas. In Dicionário Internacional da outra Economia. Coimbra, Edições Almedina.

Pegler L. (2011). Sustainable Value Chains and Labour - Linking Chain and "Inner Drivers" - From Concepts to Practice. Institute of Social Science Working Paper series, $\mathrm{n}^{\circ} 525$, pp. 1-42.

van der Ploeg J. D., Jingzhong Y., Schneider S. (2012). Rural development through the construction of new, nested, markets: comparative perspectives from China, Brazil and the European Union. The Journal of Peasant Studies, pp. 133-173, https://doi.org/10.108 0/03066150.2011.652619.

Rogez H. (2000). Açaí: preparo, composição e melhoramento da conservação. Belém, PA, EdUfpa.

Rogez H., Aguiar F. S. (2012). Contaminação da bebida açaí envolvendo o Trypanosoma cruzi. In

Rostain S. (2016). Amazonie un jardin naturel ou une forêt domestiquée. Arles, Actes Sud.

Santos J. C., Rocha C. I. L., Santos A. P., Sena A. L. S., Mattietto R. A., Elleres A. S. (2014). Descrição da cadeia produtiva do açaí na Amazônia. In Cordeiro de Santana A. (Ed.), Mercado, cadeia produtiva e desenvolvimento rural na Amazônia, Belém, Edufra, pp. 141-164.

Schneider S., Salvate N., Cassol A. (2016). Nested Markets, Food Networks, and New Pathways for Rural Development in Brazil. Agriculture, vol. 6, n 61. doi: 10.3390

Schneider S., Gazolla M. (2017). Cadeias curtas e redes agroalimentares alternativas. In Gazolla M., Schneider S. (Eds.). Cadeias curtas e redes agroalimentares alternativas: negócios e mercados da agricultura familiar, Porto Alegre, Editora UFRGS, pp. 9-24. 
RECHERCHE

Shackleton S., Shanley P., Ndoye O. (2007). Invisible but viable: Recognising local markets for non-timber forest products, The International Forestry Review, vol. 3, n 9 , pp. 697-712, Retrieved from http://www. jstor.org/stable/43739712

de Sousa F. F., Vieira-da-Silva C., Bezerra Barros F. (2018). The (in)visible market of miriti (Mauritia flexuosa L.f.) fruits, the "winter acai", in Amazonian riverine communities of Abaetetuba, Northern Brazil. Global Ecology and Conservation, $\mathrm{n}^{\circ} 14$, https://doi.org/10.1016/j.gecco.2018. e00393.

Southgate D. (1998). Tropical forest conservation: an economic assessment of the alternatives in Latin America. New York, Oxford University Press, p. 188.
Steward A. (2013). Reconfiguring agrobiodiversity in the Amazon estuary: market integration, the açaí trade and smallholders' management practices in Amapá, Brazil. Human Ecology, vol. 41, n ${ }^{\circ}$ 6, pp. 827-840.

Ticktin T. (2004). The ecological implications of harvesting non-timber forest products. Journal of Applied Ecology, $\mathrm{n}^{\circ}$ 41, pp. 11-21. doi:10.1111/j.1365-2664.2004.00859.x

Walter S., Cole D., Kathe W., Lovett P., Soldan M. P. (2003). Impact of certification on the sustainable use of NWFP: Lessonslearnt from three case studies, International Conference on Rural Livelihoods, Forests and Biodiversity, Mai, Bonn, pp. 19-23. En ligne : http://www.fao.org/forestry/foris/pdf/ NWFP/CIFOR_pres.pdf.

\section{ANNEXES}

Tableau 1. Caractéristiques des transformateurs de l'échantillon

\begin{tabular}{|c|c|c|c|c|c|c|}
\hline Type & $\begin{array}{c}\mathrm{Nb} \\
\text { employés }\end{array}$ & Ancienneté & Circuit & Lieux de vente & Produit vendu & $\begin{array}{l}\text { Type } \\
\text { d'açaí }\end{array}$ \\
\hline $\begin{array}{l}\text { Bio et équitable } \\
\text { BE1 }\end{array}$ & 180 & 10 & Export & $\begin{array}{l}\text { Supermarchés, } \\
\text { Points de vente }\end{array}$ & $\begin{array}{l}\text { Pulpe congelée, } \\
\text { Sorbets, etc. }\end{array}$ & Populaire \\
\hline $\begin{array}{l}\text { Bio et équitable } \\
\text { BE } 2\end{array}$ & $70-230$ & 11 & Export & grossiste & Pulpe congelée & Populaire \\
\hline $\begin{array}{l}\text { Bio " locale " } \\
\text { B1 }\end{array}$ & 46 & 17 & Export & grossiste & Pulpe congelée & Moyen \\
\hline $\begin{array}{l}\text { Générique } \\
\text { " santé " } \\
\text { G2 }\end{array}$ & $40-100$ & 20 & Export & grossiste & Pulpe congelée & $\begin{array}{c}\text { Populaire, } \\
\text { Moyen, } \\
\text { épais }\end{array}$ \\
\hline $\begin{array}{l}\text { Intermédiaire } \\
\text { I } 1\end{array}$ & 160 & 56 & $\begin{array}{l}\text { Métropole } \\
\text { de Belém }\end{array}$ & $\begin{array}{l}14 \text { points } \\
\text { de vente }\end{array}$ & Crème glacée & - \\
\hline $\begin{array}{l}\text { Intermédiaire } \\
12\end{array}$ & 45 & 12 & $\begin{array}{l}\text { Ville de } \\
\text { Belém }\end{array}$ & 3 restaurants & $\begin{array}{l}\text { Pulpe fraîche } \\
\text { et congelée }\end{array}$ & Moyen \\
\hline $\begin{array}{l}\text { Local artisanal } \\
\text { L1 }\end{array}$ & 10 & 15 & $\begin{array}{l}\text { Ville de } \\
\text { Belém }\end{array}$ & $\begin{array}{l}\text { Vente directe } \\
\text { et grossiste }\end{array}$ & Pulpe fraîche & Moyen \\
\hline $\begin{array}{l}\text { Local artisanal } \\
\text { L2 }\end{array}$ & 4 & 30 & $\begin{array}{l}\text { Ville de } \\
\text { Belém }\end{array}$ & Vente directe & Pulpe fraîche & Moyen \\
\hline $\begin{array}{l}\text { Local artisanal } \\
\text { L3 }\end{array}$ & 20 & 30 & $\begin{array}{l}\text { Ville de } \\
\text { Belém }\end{array}$ & $\begin{array}{c}\text { Vente directe } \\
\text { Pulpe et négociant }\end{array}$ & $\begin{array}{l}\text { Fruits et pulpe } \\
\text { fraîche }\end{array}$ & Moyen \\
\hline $\begin{array}{l}\text { Local "gourmet" } \\
\text { L4 }\end{array}$ & 10 & 40 & $\begin{array}{l}\text { Ville de } \\
\text { Belém }\end{array}$ & Vente directe & Pulpe fraîche & $\begin{array}{l}\text { Moyen, } \\
\text { spécial }\end{array}$ \\
\hline
\end{tabular}


L'açaí en Amazonie

Tableau 2. Critères de qualité cités par les transformateurs

\begin{tabular}{lcccccccc}
\hline & BE 2 & G2 & BL1 & I1 & I2 & L1 & L2 & L4 \\
\hline Couleur & $\mathrm{X}$ & $\mathrm{X}$ & $\mathrm{X}$ & $\mathrm{X}$ & $\mathrm{X}$ & $\mathrm{X}$ & $\mathrm{X}$ & $\mathrm{X}$ \\
Saveur & & $\mathrm{X}$ & $\mathrm{X}$ & $\mathrm{X}$ & $\mathrm{X}$ & $\mathrm{X}$ & & $\mathrm{X}$ \\
Rendement & $\mathrm{X}$ & & $\mathrm{X}$ & & & $\mathrm{X}$ & & $\mathrm{X}$ \\
Odeur & & & & $\mathrm{X}$ & & $\mathrm{X}$ & & $\mathrm{X}$ \\
Propreté & & & & & & & $\mathrm{X}$ & \\
"Origine " & & & $\mathrm{X}$ & & & & & \\
\hline
\end{tabular}

Source : enquêtes de terrain.

Tableau 3. Pratiques permettant d'atteindre la qualité recherchée

\begin{tabular}{|c|c|c|c|c|c|c|c|c|c|c|}
\hline & & BE 2 & G2 & BL1 & 11 & 12 & $\mathrm{~L} 1$ & L2 & L3 & $L 4$ \\
\hline \multicolumn{11}{|c|}{ Cueillette } \\
\hline 1 & $\begin{array}{l}\text { Battre les fruits sur } \\
\text { une bâche }\end{array}$ & $x$ & & $\mathrm{X}$ & & $x$ & $x$ & $x$ & $x$ & \\
\hline 2 & $\begin{array}{l}\text { Ne pas « griffer » les } \\
\text { fruits }\end{array}$ & & & & & & & & & $x$ \\
\hline 3 & $\begin{array}{l}\text { Cueillir les fruits à } \\
\text { maturité }\end{array}$ & $x$ & $x$ & & & & & $x$ & $x$ & $\mathrm{x}$ \\
\hline 4 & $\begin{array}{l}\text { Retirer les débris } \\
\text { végétaux }\end{array}$ & $x$ & $x$ & $\mathrm{X}$ & & $x$ & $x$ & $x$ & $x$ & \\
\hline \multicolumn{11}{|c|}{ Transport } \\
\hline 5 & Minimiser le temps & & $x$ & $\mathrm{X}$ & & $\mathrm{x}$ & & $x$ & $x$ & $x$ \\
\hline 6 & Ne pas « mouiller » & & $x$ & $x$ & & $x$ & & $x$ & $x$ & \\
\hline 7 & Manière de dépulper & & & & & & & & & $x$ \\
\hline
\end{tabular}

Source : enquêtes de terrain. 